\title{
The Partnered Core of a Game with Side Payments \\ by
}

Philip J. Reny, Eyal Winter, and Myrna Wooders

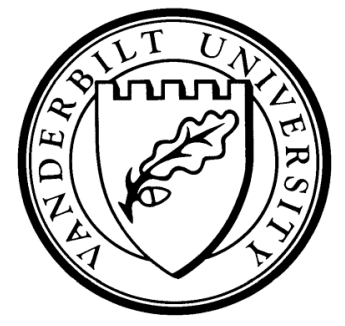

Working Paper No. 09-W17

October 2009

\section{DEPARTMENT OF ECONOMICS \\ VANDERBILT UNIVERSITY \\ NASHVILLE, TN 37235}

www.vanderbilt.edu/econ 


\title{
The Partnered Core of a Game With Side Payments*
}

\author{
Philip J. Reny \\ University of Chcago Hebrew University \\ Myrna Wooders \\ Vanderbilt University \\ Keywords: partnered core, separating collections of coalitions, \\ cooperative games \\ JEL: $\mathrm{C} 71$
}

\begin{abstract}
An outcome of a game is partnered if there are no asymmetric dependencies between any two players. For a cooperative game, a payoff is in the partnered core of the game if it is partnered, feasible and cannot be improved upon by any coalition of players. We show that the relative interior of the core of a game with side payments is contained in the partnered core. For quasi-strictly convex games the partnered core coincides with the relative interior of the core. When there are no more than three partnerships, the sums of the payoffs to partnerships are constant across all core payoffs. When there are no more than three players, the partnered core satisfies additional properties.
\end{abstract}

*This research was initiated in 1991 when the authors were guests of Sonderforschungsbereich 303 at the University of Bonn and appeared in several academic working paper series (see references). The hospitality and support of Sonderforschungsbereich 303 and the University of Bonn is gratefully acknowledged. The authors are also indebted to the Social Sciences and Humanities Research Council of Canada for support which made this collaboration possible. We thank Elaine Bennett, Arja Turunen-Red, Preston McAfee, Larry Samuelson, and Arthur Robson for helpful comments. 


\section{The Partnership Property}

An intuitively appealing property of a solution for a game-theoretic model is symmetry of dependencies. If one player needs the cooperation of a second player to achieve his payoff but the second player has alternative coalitions not including the first player then the second player is in an apparently stronger position. This is an asymmetric dependency. In such a case we may expect the stronger player to attempt to use this dependency to increase his own payoff at the expense of the more dependent player. A solution is said to have the partnership property if there are no asymmetric dependencies. The partnership property has a long history in cooperative game theory. It appeared in Maschler and Peleg $(1966,1967)$, Peleg (1968) and Maschler, Peleg, and Shapley (1972) in their impressive study of the kernel and separating (partnered) collections of coalitions. The partnership property on the domain of undominated payoffs was introduced in Albers $(1974,1979)$, and further studied in Selten (1981), Bennett (1980,1983), Bennett and Zame (1988), Winter (1989) and Moldovanu and Winter (1994). More recently, the partnership property on the set of undominated payoffs was studied in Reny and Wooders (1996). Partnership has also been used to characterize price-taking economic equilibrium in Bennett and Zame (1998) and no-arbitrage equilibrium in Page and Wooders (1996) and in a study of commonwealths in Reny and Wooders (1996). ${ }^{1}$ In the current paper we study the partnered core of a game with side payments. This framework has the advantage of tractability and allows additional results not obtainable in the case of games without side payments.

Two familiar examples illustrate the appeal of the partnership property. First, consider a two-person bargaining problem of dividing a dollar. Any division of the total payoff between the two players is in the core. To achieve the payoff $(\$ .50, \$ .50)$, each player must have the cooperation of the other player; thus the players are partnered. By contrast, the payoff $(\$ 1.00, \$ .00)$ is not partnered and the coalition consisting of the two players is not compelled to form. One player has an alternative coalition - himself alone- in which he can realize his part of the payoff; the other player does not have such an option. In other situations, no payoff in the core exhibits the instability demonstrated by the payoff $(\$ 1.00, \$ .00)$ for the divide-the-dollar game. For example, in a game with one seller who owns one indivisible unit of a good and two potential buyers, each of whom is willing to pay a dollar for the good, the only payoff in the core is $\$ 1.00$ to the seller

\footnotetext{
${ }^{1}$ The partnership property also is related to the KKMS Theorem; see Reny and Wooders (1998) and Kannai and Wooders (2000).
} 
and $\$ 0.00$ to each of the two buyers. If the player who actually buys the good attempts to obtain a larger share of the surplus, the seller has the possibility of approaching the other potential buyer and coming to some new agreement with that buyer. The seller is not dependant on either buyer, and each buyer receives only his individually rational payoff. Thus, there are no asymmetric dependences between any pair of players.

Following Maschler and Peleg $(1966,1967)$ we formulate the partnership property directly on collections of coalitions. A collection of coalitions has the partnership property if for each player $i$, whenever $j$ is a member of all the coalitions containing player $i, i$ is a member of all the coalitions containing player $j$. A largest set of players whose members are in all the same coalitions in the collection is called a partnership and the players in the partnership are called partners. A collection is minimally partnered if the only partner of a player is the player himself. It is maximally partnered if the partners of each player include all other players.

A payoff for a game is partnered if the collection of coalitions that can afford that payoff for their members has the partnership property. We define the partnered core as the set of payoffs that are partnered, feasible, and undominated. We show that the partnered core contains the relative interior of the core and present an example in which the containment is strict. Thus, whenever the core is nonempty, almost all, but not necessarily all, core payoffs are partnered. For quasi-strictly convex games, however, we prove that the set of undominated payoffs with the partnership property coincides with the interior of the core of the game.

For games with no more than three partnerships, we provide additional results on the partnership property of the core. In particular, the collection of partnerships as well as the payoff to any particular partnership is constant across all payoffs in the relative interior of the core. Also, when the total number of players is less than or equal to three, we show that the partnered core coincides with the core's relative interior and that all core payoffs are minimally partnered - no one is dependent on anyone - if and only if the core is a singleton set.

We employ the framework of a game in characteristic form with side payments. This framework does not directly treat negotiations between players. Partnered payoffs, however, have been shown to arise as outcomes of bargaining. In a noncooperative setting, Selten (1981) shows that the subgame perfect equilibrium payoffs of a coalitional bargaining game are partnered. We discuss the relationship of our framework and results to games with coalition structures in Section 6. We 
argue that partnerships are game-theoretically important components of coalition structure games, as in Aumann and Dreze (1974). In particular, we emphasize the distinction between partnerships and coalition structures required to achieve gains to the collective activities described by the characteristic function. We discuss related literature more generally in Section 7.

\section{Games}

A game (in characteristic form) is a pair $(N, v)$ where $N=\{1, \ldots, n\}$ is a finite set of players and $v$ is a function from $2^{N}$ to $\mathbf{R}_{+}$with $v(\emptyset)=0$. A nonempty subset $S$ of $N$ is called a coalition.

A payoff for a game $(N, v)$ is a vector $x$ in $\mathbf{R}^{N}$. Let $S \subseteq N$ and define $x(S)=\sum_{i \in S} x_{i}$. A payoff $x$ is feasible if $x(N)=v(N)$. A payoff $x$ is undominated if $x(S) \geq v(S)$ for all coalitions $S \subset N$. The coalitions $S$ with $v(S) \geq x(S)$, denoted by $\mathcal{S}(x)$, are said to support the payoff $x$. The coalitions in $\mathcal{S}(x)$ that contain the $i^{\text {th }}$ player are denoted by $\mathcal{S}_{i}(x)=\{S \in \mathcal{S}(x): i \in S\}$. The core of a game $(N, v)$ is denoted by $C(N, v)$ and defined by

$$
C(N, v)=\left\{x \in \mathbf{R}^{N}: x \text { is a feasible and undominated payoff for }(N, v)\right\} .
$$

We denote the relative interior of the core by $\operatorname{riC}(N, v)$.

\section{Partnership}

Let $N$ be a finite set of players and let $P$ be a collection of subsets of $N$. For each $i$ in $N$ let

$$
P_{i}=\{S \in P: i \in S\} .
$$

We say that $P$ has the partnership property (for $N$ ) if for each $i$ in $N$ the set $P_{i}$ is nonempty and for each pair of players $i$ and $j$ in $N$ the following requirement is satisfied:

$$
\text { if } P_{i} \subset P_{j} \text { then } P_{j} \subset P_{i} \text {. }
$$

That is, if all the coalitions in $P$ that contain player $i$ also contain player $j$ then all the coalitions that contain $j$ also contain $i{ }^{2}$ We say that players $i$ and $j$

\footnotetext{
${ }^{2}$ For the reader familiar with these works, we note here that partnered collections of coalitions are called "separating collections" in Maschler and Peleg $(1966,1967)$, and Maschler, Peleg, and Shapley (1971). See Section 7 for further discussion.
} 
are partners (or that $i$ is partnered with $j$ ) if $P_{i}=P_{j}$. Clearly, the relation "is partnered with" is an equivalence relation. Consequently, let $\mathcal{P}[i]$ denote the equivalence class containing $i^{\prime} s$ partners, and call $\mathcal{P}[i]$ a partnership. For any collection $P$ with the partnership property we say that $P$ is minimally (maximally) partnered if $\mathcal{P}[i]=\{i\}$ for each player $i(\mathcal{P}[i]=N)$. While our results will focus on feasible payoffs, the partnership property is a property of collections of sets and does not involve any feasibility requirements.

Let $(N, v)$ be a game and let $x$ be a payoff for $(N, v)$. The payoff $x$ is called a partnered payoff if the collection of coalitions that support $x, \mathcal{S}(x)$, has the partnership property. In this case we denote the partnership containing player $i$ by $\mathcal{P}_{x}[i]$ and the collection of partnerships induced by $x$ by $\mathcal{P}_{x}$. The payoff $x$ is minimally partnered (maximally partnered) if it is partnered and if the set of supporting coalitions $\mathcal{S}(x)$ is minimally (maximally) partnered. Note that it is not required that partnered payoffs be feasible.

The partnered core is denoted by $C^{*}(N, v)$ and is defined by

$$
C^{*}(N, v)=\{x \in C(N, v): x \text { is a partnered payoff }\} .
$$

Our first Theorem establishes the close connection between the core and the partnered core for games with side payments; the relative interior of the core is contained in the partnered core. The Theorem also states that the set of partners of a player (and hence the partition of $N$ into partnerships) is unchanged over all points in the relative interior of the core.

Theorem 1. Let $(N, v)$ be a game. Then

$$
\operatorname{riC}(N, v) \subseteq C^{*}(N, v)
$$

and for all $x, y$ in $\operatorname{riC}(N, v)$,

$$
\mathcal{P}_{x}[i]=\mathcal{P}_{y}[i]
$$

Note that since $\operatorname{riC}(N, v) \subseteq C^{*}(N, v)$ the partnerships $\mathcal{P}_{x}[i]$ and $\mathcal{P}_{y}[i]$ are welldefined. The result that $\mathcal{P}_{x}[i]$ is equal to $P_{y}[i]$ is a consequence of the fact that the set of supporting coalitions is constant over all points in the relative interior of the core. The proof of Theorem 1 requires some linear programming results. These, and the proof of the Theorem, are contained in the next section. ${ }^{3}$

\footnotetext{
${ }^{3}$ The first part of our Theorem is also proved by Albers (1979, Lemma 3.3 ). Both proofs follow the same line of argument; ours, however, provides more detail - specifically, Lemmas 1 to 3. It follows from Albers' Lemma that the set of undominated and partnered payoffs is nonempty. The latter result is also proved in Bennett (1983).
} 
Combining Theorem 1 with the Bondareva (1963) and Shapley (1967) Theorem that a game with side payments has a nonempty core if and only if it is balanced leads to the Corollary below. We refer the reader unfamiliar with the concept to Section 4 for the definition of a balanced game.

Corollary 1 . The partnered core of a balanced game is nonempty.

The following example shows that the containment expressed in Theorem 1 may be strict.

Example $\mathbf{1}^{4}$ : Let $(N, v)$ be a game where $N=\{1,2,3,4\}$,

$$
\begin{gathered}
v(N)=4, \\
v(\{1,3\})=2, \\
v(1,4\})=2, \\
v(\{2,3\})=2, \\
v(\{2,4\})=2, \text { and } \\
v(S)=0 \text { for all other coalitions } S \subset N .
\end{gathered}
$$

The core consists of the set

$$
\left\{x: x_{1}=2-x_{4}, x_{2}=2-x_{3}, x_{3}=x_{4}, x_{4} \in[0,2]\right\} .
$$

Note that for all points in the core the constraints associated with the coalitions

$$
\{1,3\},\{1,4\},\{2,3\} \text {, and }\{2,4\}
$$

are binding. Consequently (see Lemma 3 in the next section), the solution to the equations associated with these coalitions characterizes the relative interior of the core. Thus, every payoff in the relative interior is minimally partnered. This implies every core payoff is minimally partnered. Therefore $(0,0,2,2)$, in the boundary of the core and not in $\mathrm{riC}(N, v)$, is partnered.

Although all core payoffs may be minimally partnered, as in the above example, our next Theorem shows that not all core payoffs can be maximally partnered. Indeed the Theorem provides a somewhat more general result on the partnership property of the core.

\footnotetext{
${ }^{4}$ This example is based on an observation due to Preston McAfee
} 
Theorem 2: Let $(N, v)$ be a balanced game. Then there is a payoff in $C(N, v)$ that is either minimally partnered or not partnered.

Proof of Theorem 2: Let $x^{*}$ be any extreme point of the core. If $x^{*}$ is not partnered, we're done. So, suppose $x^{*}$ is partnered and not minimally partnered. Then there is a pair of distinct players $i$ and $j$ who are partners. Consequently, $\mathcal{S}_{i}\left(x^{*}\right)=\mathcal{S}_{j}\left(x^{*}\right)$, so that we can slightly increase the payoff to $i$ (or $j$ ) and correspondingly decrease the payoff to $j$ (or $i$ ) while not violating any of the constraints given by $\mathcal{S}\left(x^{*}\right)$. Thus, there is more than one solution to the equations given by

$$
x(S)=v(S) \text { for all } S \text { in } \mathcal{S}\left(x^{*}\right) .
$$

But this contradicts the extremal property of $x^{*}$, (i.e., $x^{*}$, being extreme, must be the unique solution to the set of binding constraints).

Remark: The conclusion of Theorem 2 is tight. In Example 1 all payoffs (and therefore all extreme payoffs) in the core are minimally partnered. An example of a game in which no extreme payoff in the core is partnered and no payoff in the core is minimally partnered is a two-person bargaining game.

The following Corollary is immediate from Theorems 1 and 2 .

Corollary 2: $C(N, v)=\{x\}$ implies $x$ is minimally partnered.

For games with empty cores there exist undominated partnered payoffs. For example, all payoffs in the relative interior of the core of the balanced cover game are both undominated and partnered. These payoffs however are not necessarily feasible, as illustrated by the following example.

Example 2: A three-person simple majority game. Let $N=\{1,2,3\}$ and let

$$
\begin{gathered}
\qquad v(N)=1, \\
v(\{i, j\})=1 \text { for each pair of distinct players } i \text { and } j,
\end{gathered}
$$

and

$$
v(\{i\})=0 \text { for all } i .
$$

The payoff $x=\left(\frac{1}{2}, \frac{1}{2}, \frac{1}{2}\right)$ is clearly undominated and it is also partnered since the collection of supporting sets $\{\{1,2\},\{2,3\},\{1,3\}\}$ is partnered. It is not, however, feasible. 
For a class of convex games the set of undominated and partnered payoffs coincides with the relative interior of the core. Let $(N, v)$ be a game. The game is quasi-strictly convex if for any pair of non-nested sets $S$ and $T$ contained in $N$,

$$
v(S)+v(T) \leq v(S \cup T)+v(S \cap T)
$$

with strict inequality if $S \cup T=N$. Thus, the game is convex and the gains to forming the grand coalition are strictly positive. Note that all strictly convex games are quasi-strictly convex, but the converse is not true. An example of a quasi-strictly convex game which is not strictly convex is a pure bargaining game, where $v(S)=0$ for all $S \neq N$ and $v(N)=1$.

Theorem 3: Suppose that $(N, v)$ is a quasi-strictly convex game. Then a payoff $x$ is undominated and partnered if and only if $x$ is in $\operatorname{riC}(N, v)$.

Proof of Theorem 3: From Theorem 1 it follows that if a payoff $x$ is in $\operatorname{riC}(N, v)$ then $x$ is partnered. To prove the Theorem it now suffices to show that if a payoff $x$ is partnered and undominated then it is in $\operatorname{riC}(N, v)$. And to show that $x \in \operatorname{riC}(N, v)$ it suffices to show that (i) $x(N)=v(N)$ and (ii) $x(S)>v(S)$ for all coalitions $S \neq N$. Since $x$ is partnered, (ii) implies (i). (Partnership of the payoff $x$ implies that for each player $i$ there is at least one coalition containing player $i$ supporting the payoff $x$. Since (ii) implies that no coalition smaller than $N$ supports $x, N$ must support $x$. Finally, since $x$ is undominated we must then have $x(N)=v(N)$.) Hence it suffices to show (ii). So, proceed by assuming that (ii) is false. In particular, assume that there is some coalition $S \neq N$ such that $x(S)=v(S)$. (Recall that $x(S)<v(S)$ is impossible since $x$ is undominated.) Choose $i \in S$ and $j \notin S$. Since $x$ is partnered, there exists $T \subset N$ with $j \in T$, $i \notin T$, and $x(T)=v(T)$. Since $S$ and $T$ are non-nested we then have by quasistrict convexity that

$$
\begin{gathered}
x(S \cup T)+x(S \cap T) \\
=x(S)+x(T)=v(S)+v(T) \leq v(S \cup T)+v(S \cap T) .
\end{gathered}
$$

Hence, $x(S \cup T)=v(S \cup T)$ and $x(S \cap T)=v(S \cap T)$. We have therefore shown the following: for each strict subset $S$ of $N$ satisfying $x(S)=v(S)$ there is a non-nested strict subset $T$ of $N$ satisfying $x(T)=v(T), x(S \cup T)=v(S \cup T)$ and $x(S \cap T)=v(S \cap T)$. Let $\mathcal{S}$ denote the non-empty, finite set of all such $(S, T)$ pairs. Choose $\left(S^{*}, T^{*}\right) \in \mathcal{S}$ such that $S^{*} \cup T^{*}$ is maximal with respect to set inclusion. 
Now since $x\left(S^{*}\right)=v\left(S^{*}\right), x\left(T^{*}\right)=v\left(T^{*}\right), x\left(S^{*} \cup T^{*}\right)=v\left(S^{*} \cup T^{*}\right)$, and $x\left(S^{*} \cap T^{*}\right)=v\left(S^{*} \cap T^{*}\right)$, it follows that $v\left(S^{*}\right)+v\left(T^{*}\right)=v\left(S^{*} \cup T^{*}\right)+v\left(S^{*} \cap T^{*}\right)$. Consequently by the quasi-strict convexity of $(N, v)$ it must be the case that $S^{*} \cup T^{*} \subset N$, and $S^{*} \cup T^{*} \neq N$.

Let $Q=S^{*} \cup T^{*}$. Since $x(Q)=v(Q)$ there is a non-nested subset $R$ of $N$ satisfying $x(R)=v(R)$ and $x(R \cup Q)=v(R \cup Q)$. Hence, $(Q, R) \in \mathcal{S}$. But this contradicts the maximality of $S^{*} \cup T^{*}=Q \subset Q \cup R$ and $Q \neq Q \cup R$.

Corollary 3: If $(N, v)$ is a quasi-strictly convex game then the interior of the core, $\operatorname{int} C(N, v)$, is nonempty and $x$ is partnered if and only if $x$ is maximally partnered. ${ }^{5}$

Proof of Corollary 3: Choose $x^{0} \in \operatorname{riC}(N, v)$. Then by the proof of Theorem $3, x^{0}(N)=v(N)$ and $x^{0}(S)>v(S)$ for all proper subsets $S$ of $N$ (since $x^{0}$ is partnered). Hence $x^{0} \in \operatorname{int} C(N, v)$, and the first part is proven. The second part follows since the fact that $x^{0}$ is partnered means that (as before) the only binding constraint is $x^{0}(N)=v(N)$. Consequently, $x^{0}$ is maximally partnered.

\section{Some Results on the Core and Linear Programming}

To prove Theorem 1 and the results in the following section, we require some linear programming results. First, for the convenience of the reader, we present the well-known (c.f., [17] p. 154) linear programming characterization of the core.

A game $(N, v)$ has a nonempty core if and only if the following linear program

$$
\begin{gathered}
\operatorname{minimize} \sum_{i=1}^{n} x_{i}=z \\
\text { subject to } \sum_{i \in S} x_{i} \geq v(S) \text { for all } S \subseteq N
\end{gathered}
$$

has a minimum $z^{*} \leq v(N)$. Any minimizing $x$ lies in the core. Conversely, if $x \in$ $C(N, v)$ then $x(S) \geq v(S)$ for all coalitions $S$; moreover, $\sum_{i=1}^{n} x_{i}=v(N)$. Thus, the minimum $z^{*}$ must satisfy $z^{*} \leq v(N)$.

\footnotetext{
${ }^{5}$ Because every core payoff must be feasible, we view the core as a subset of $R^{n-1}$. Consequently, the interior of the core is nonempty if and only if the core has full dimension, $n-1$.
} 
Consider the dual program to (4.1):

$$
\begin{gathered}
\operatorname{maximize} \sum_{i=1}^{n} \omega_{S} v(S)=q, \\
\text { subject to } \sum_{\substack{S \\
i \in S \subseteq N}} \omega_{S}=1 \text { for all } i \in N, \text { and } \\
\omega_{S} \geq 0 \text { for all } S \subseteq N .
\end{gathered}
$$

Program (4.1) is feasible if and only if (4.2) is feasible and, in this case, the minimum $z^{*}$ equals the maximum $q^{*}$. Thus, $C(N, v) \neq \emptyset$ if and only if $q^{*} \leq v(N)$.

The above is the proof of the Bondareva (1963) and Shapley (1967) Theorem that a game with side payments has a nonempty core if and only if the game is balanced. Let $\beta$ be a collection of nonempty subsets of $N$. We say that $\beta$ is balanced if there exist positive real numbers, called balancing weights, $\omega_{S}$ for $S \in \beta$ such that

$$
\sum_{\substack{S \in \beta \\ i \in S}} \omega_{S}=1 \text { for all } i \in N .
$$

A game $(N, v)$ is balanced if for every balanced collection $\beta$ with balancing weights $\omega_{S}$ for $S \in \beta$,

$$
\sum_{S \in \beta} \omega_{S} v(S) \leq v(N) .
$$

The balancedness condition (4.4) characterizing games with nonempty cores will be used in the proof of Lemma 3 below.

Throughout the remainder of this section $A$ and $B$ denote $m \times n$ matrices and $b, c, \ldots$ denote vectors in $\mathbf{R}^{n}$.

Lemma 1: Let $\mathcal{C}$ be the convex subset of $\mathbf{R}^{n}$ defined by

$$
\mathcal{C}=\left\{x \in \mathbf{R}^{n}: A x \geq b, B x \geq c\right\} .
$$

Further, assume that $A x^{0}=b$ and $B x^{0}>c$ for some $x^{0} \in$ riC, the relative interior of $\mathcal{C}$. Then

$$
\text { riC }=\left\{x \in \mathbf{R}^{n}: A x=b, B x>c\right\} .
$$

Proof of Lemma 1: Let $X=\left\{x \in \mathbf{R}^{n}: A x=b, B x>c\right\}$. 
Part 1: To show that $\mathrm{riC} \subset X$ we first show that for all $z \in \mathcal{C}, A z=b$. So, choose any $z \in \mathcal{C}$. Since $x^{0} \in \operatorname{riC}$, there exists $\alpha \in(0,1]$ and $y \in \mathcal{C}$ such that $x^{0}=\alpha z+(1-\alpha) y$. But $A z \geq b, A y \geq b$, and $\alpha \in(0,1]$, together with $A x^{0}=b$, imply that $A z=b$.

Now choose any $x^{*} \in \mathrm{riC}$. Then there exist $\alpha \in(0,1]$ and $y \in \mathcal{C}$ such that $x^{*}=\alpha x^{0}+(1-\alpha) y$. Hence, $A x^{*}=b$ and $B x^{*}>c$.

Part 2: To show that $X \subset$ riC let $L^{*}$ be the smallest linear subspace such that $\mathcal{C} \subset L^{*}+x^{0}$. Recall that $x \in \operatorname{riC}$ if (and indeed only if) for all $y \in L^{*}, x+\alpha y \in \mathcal{C}$ for all $\alpha$ sufficiently small (Rockafellar (1970), p. 44). Consequently, it is enough to show that $L^{*} \subset\{x: A x=0\}$.

So, let $\tilde{L}=\{x: A x=0\}$. Since $\tilde{L}$ is a linear subspace, it suffices, by the definition of $L^{*}$ to show that $\mathcal{C} \subset \tilde{L}+x^{0}$. So, choose $x \in \mathcal{C}$. Then as shown in the first part of the proof, A $x=b$. Since A $x^{0}=b$, we have $y=x-x^{0} \in \tilde{L}$, that is, $x=y+x^{0} \in \tilde{L}+x^{0}$.

Remark: Consider the system of linear inequalities $A x \leq b$, and denote its (convex) set of solutions by $\mathcal{C}$. Suppose $\mathcal{C} \neq \emptyset$ and choose $x^{0} \in \operatorname{riC}$. Let $E=\{i$ : $\left.A_{i} x^{0}=b_{i}\right\}$, where $A_{i}$ denotes the $i^{t h}$ row of A. Then by Part 1 of the proof above, for every $i$ in $E, A_{i} x \leq b_{i}$ is binding in every solution $x \in \mathcal{C}$. Moreover, because these are the only inequalities binding for $x^{0}$, these are precisely the inequalities that must be binding in every solution. Hence, by Lemma $1, x^{*}$ is in $r i \mathcal{C}$ if and only if those constraints binding for $x^{*}$ are binding in every solution.

The next Lemma relates constraints that are binding in every solution in a linear programming problem to positive values of solutions to the corresponding dual variables. The Lemma is the converse to the complementary slackness Theorem.

Lemma 2: If the first constraint is binding in every solution to the primal problem,

$$
\begin{gathered}
\text { maximize } c \cdot x \\
\text { subject to } A \cdot x \leq b, x \geq 0,
\end{gathered}
$$

then there exists a solution to the dual problem,

$$
\begin{gathered}
\operatorname{minimize} b \cdot y \\
\text { subject to } A^{T} y \geq c, y \geq 0,
\end{gathered}
$$

in which the first dual variable is strictly positive.

Proof. See Schrijver (1990), p.95. 
Remark: Let $\mathcal{C}$ denote the set of solutions to the linear programming problem (4.5). Our first two Lemmas show that for every point $x^{*}$ in riC there is a solution to the dual problem (4.6) with the property that every dual variable corresponding to a constraint that is binding at $x^{*}$ has a positive weight.

Our next Lemma relates balanced collections of coalitions to the relative interior of the core.

Let $(N, v)$ be a game. Define the collection of sets $\mathcal{B}$ by

$$
\begin{gathered}
\mathcal{B}=\{S \subset N: \text { for every solution } x \text { to the program (4.1) } \\
\text { the constraint } x(S) \geq v(S) \text { is binding }\} .
\end{gathered}
$$

Lemma 3: Let $x^{*} \in C(N, v)$. Then $\left\{S: x^{*}(S)=v(S)\right\}$ is balanced if and only if $x^{*} \in \operatorname{riC}(N, v)$.

Proof. Let $x^{*} \in C(N, v)$. Assume $\mathcal{S}\left(x^{*}\right)=\left\{S: x^{*}(S)=v(S)\right\}$ is balanced. Let $\left(\delta_{S}\right)_{S \in \mathcal{S}\left(x^{*}\right)}$ be a collection of balancing weights for $\mathcal{S}\left(x^{*}\right)$. Since $x^{*} \in C(N, v)$,

$$
v(N)=x^{*}(N)=\sum_{S \in \mathcal{S}\left(x^{*}\right)} \delta_{S} x^{*}(S)=\sum_{S \in \mathcal{S}\left(x^{*}\right)} \delta_{S} v(S) .
$$

Consequently, $\left(\delta_{S}\right)$ is a solution to the dual programming problem (4.2). By the definition of balancedness, $\delta_{S}>0$ for each $S \in \mathcal{S}\left(x^{*}\right)$. It follows that $\mathcal{S}\left(x^{*}\right) \subseteq \mathcal{B}$. For suppose not. Then there is a solution $x^{0}$ to the primal problem and $S \in \mathcal{S}\left(x^{*}\right)$ such that $x^{0}(S)>v(S)$. This implies that $v(N)=x^{0}(N)=\sum_{S \in \mathcal{S}\left(x^{*}\right)} \delta_{S} x^{0}(S)>$ $\sum_{S \in \mathcal{S}\left(x^{*}\right)} \delta_{S} v(S)=v(N)$ since $x^{0} \in C(N, v)$ and $x^{0}(S)>v(S)$ for at least one coalition $S$ in $\mathcal{S}\left(x^{*}\right)$, yielding a contradiction. Moreover, $x^{*} \in C(N, v)$ implies $\mathcal{B} \subseteq \mathcal{S}\left(x^{*}\right)$. We conclude that $\mathcal{S}\left(x^{*}\right)=\mathcal{B}$ and so by the remark following Lemma $2, x^{*} \in \operatorname{riC}(N, v)$.

Let $x^{*}$ be a payoff in $\operatorname{riC}(N, v)$. From the remark following Lemma 2 , there is a solution to the dual problem (4.6) with the property that every dual variable corresponding to a primal constraint that is binding at $x^{*}$ is positive. Thus, the collection of supporting coalitions $\mathcal{S}\left(x^{*}\right)=\left\{S \subset N: x^{*}(S)=v(S)\right\}$ is balanced.

Proof of Theorem 1: Let $x$ be a payoff in $\operatorname{riC}(N, v)$. From Lemma 3 the collection of supporting coalitions $\mathcal{S}(x)$ is balanced. Consequently, $\Sigma_{S \in S_{i}(x)} \omega_{S}=$ 1 and $\Sigma_{S \in S_{j}(x)} \omega_{S}=1$ for any set of balancing weights $\left\{\omega_{S}\right\}_{S \in S(x)}$. Hence it is impossible that $\mathcal{S}_{i}(x) \subset \mathcal{S}_{j}(x)$ and $\mathcal{S}_{i}(x) \neq \mathcal{S}_{j}(x)$. We conclude that $x$ is partnered.

The second part of the Theorem follows from the observation (see Lemma 1) that the set of binding constraints is constant over all points in $\operatorname{riC}(N, v)$. Consequently the set of partners of a player is unchanged over all points in $\operatorname{riC}(N, v)$. 
The following Lemma will be used in the next section.

Lemma 4. Suppose that $A$ is an $m \times n$ matrix consisting entirely of zeros and ones and that $n$, the number of columns, is no greater than three. If for any pair of distinct columns, $j$ and $j^{\prime}$ there is a row, $i$, of $A$ whose $j^{\text {th }}$ entry is one and whose $j^{\prime t h}$ entry is zero, then $A$ has rank $n$.

Proof: Since there are potentially eight distinct rows, it is straightforward to simply exhaust all possibilities.

\section{Games with Three or Fewer Partnerships}

In this Section we obtain some stronger results for games with no more than three partnerships. This condition is obviously satisfied if there are no more than three players; our first two results concern such games.

Theorem 4: Let $(N, v)$ be a game with $|N| \leq 3$. Then a payoff $x \in C(N, v)$ is minimally partnered if and only if $C(N, v)=C^{*}(N, v)=\{x\}$.

Proof of Theorem 4: In view of Corollary 2, it suffices to show that if a payoff $x \in C(N, v)$ is minimally partnered then $x$ is the unique core payoff. Observe that if $x \in C(N, v)$ is minimally partnered then $\{S: x(S) \leq v(S)\}$ has a balanced subset that is itself minimally partnered (this can be verified by checking all possible cases). Now, since the subset is balanced the associated constraints in problem (4.1) must hold with equality in every solution. But by Lemma 4 this balanced subset then yields a system of equations with $x$ as a unique solution since it is minimally partnered. Therefore $C(N, v)=C^{*}(N, v)=\{x\}$.

Theorem 5: Let $(N, v)$ be a game with $|N| \leq 3$. Then $x \in C(N, v)$ is partnered if and only if $x \in \operatorname{riC}(N, v)$.

Proof of Theorem 5: In view of Theorem 1, it suffices to show that if $x$ $\in C(N, v)$ is partnered then it is in $\operatorname{riC}(N, v)$. If $x \in C(N, v)$ is minimally partnered then we're done by Theorem 4 . So suppose that $x$ is partnered but not minimally partnered. Consequently there are four possibilities for $\mathcal{S}(x)$ : $\{\{1,2\},\{3\}\},\{\{1,3\},\{2\}\},\{\{1\},\{2,3\}\}$ and $\{\{1,2,3\}\}$. Clearly, each of the four possible collections of supporting coalitions for $x$ is balanced. Thus, by Lemma 3 , $x \in \operatorname{riC}(N, v)$.

Recall that all relative interior core points induce the same partnerships. Our next result states that if there are no more than three partnerships supporting 
payoffs in the relative interior of the core, then the total payoff to each partnership is constant across all payoffs in the core's relative interior.

Theorem 6: Let $x$ and $y$ be payoffs in $\operatorname{riC}(N, v)$ and define $\mathcal{P}[i]=\mathcal{P}_{x}[i](=$ $\left.\mathcal{P}_{y}[i]\right)$. Suppose that there are no more than three partnerships in the supporting collection $\mathcal{S}(x)(=\mathcal{S}(y))$. Then for each partnership $\mathcal{P}[i]$,

$$
\sum_{j \in \mathcal{P}[i]} x_{i}=\sum_{j \in \mathcal{P}[i]} y_{i}
$$

Proof of Theorem 6: Choose $x^{*} \in \operatorname{riC}(N, v)$. Consider the case in which $x^{*}$ induces three partnerships which we denote by $\mathcal{P}\left[i_{1}\right], \mathcal{P}\left[i_{2}\right], \mathcal{P}\left[i_{3}\right]$. (Other cases can be handled similarly.) Recall then that the set of supporting coalitions for $x^{*}, \mathcal{S}\left(x^{*}\right)=\left\{S: x^{*}(S)=v(S)\right\}$ enjoys the following properties: (i) If $S \in \mathcal{S}\left(x^{*}\right)$ and $S \cap \mathcal{P}\left[i_{j}\right] \neq \emptyset$ then $S \supseteq \mathcal{P}\left[i_{j}\right]$; (ii) If $\mathcal{P}\left[i_{j}\right] \neq \mathcal{P}\left[i_{k}\right]$, then there exists $S \in \mathcal{S}\left(x^{*}\right)$ such that $S \supseteq \mathcal{P}\left[i_{i}\right]$ and $S \cap \mathcal{P}\left[i_{k}\right]=\emptyset$.

Introduce three free variables $y_{1}, y_{2}$, and $y_{3}$. Then, by letting $y_{k}=x\left(\mathcal{P}\left[i_{k}\right]\right)$, for every $S \in \mathcal{S}\left(x^{*}\right)$ the associated equation $x(S)=v(S)$ can, by (i), be written as $\Sigma y_{k}=v(S)$, where the sum is taken over those $k \in\{1,2,3\}$ such that $S \supseteq \mathcal{P}\left[i_{k}\right]$. Taken together, these equations provide a linear system in the three variables $y_{1}, y_{2}$, and $y_{3}$. Write this system as $A y=w$ and note that $A$ is a matrix with three columns having, by (ii), the properties listed in the hypotheses of Lemma 4. Consequently, $A$ has rank 3 and the unique solution to the system is, say, $y_{1}^{*}, y_{2}^{*}$, and $y_{3}^{*}$. Consequently, $x^{*}\left(\mathcal{P}\left[i_{k}\right]\right)=y_{k}^{*}$ for $k=1,2,3$. Moreover, since the system $A y=w$ is independent of the chosen point in $\operatorname{riC}(N, v)$, we must have $x\left(\mathcal{P}\left[i_{k}\right]\right)=y_{k}^{*}$ for every $x \in \operatorname{riC}(N, v)$.

\section{A Remark On Coalition Structure Games}

In applications of the theory of games in characteristic form it is often assumed that a part of the problem to be solved is the division of players into groups for the purposes of joint consumption and/or production within groups, for example, the collective consumption of public goods (c.f., Wooders (1994, Section 6) for a survey of some applications). In these problems, a characteristic function, say $w$, is derived from the underlying social and economic data. The function $w$ specifies the payoff to any coalition $S$ when the members of $S$ engage in collective activities. It is frequently useful to make a distinction between the payoff to a group acting together and the payoffs realizable by a group when it is permitted to divide into 
subgroups. There may be congestion, for example, in the consumption of public facilities, so that a number of groups may each have their own facilities. Then a superadditive characteristic function $v$, called the superadditive cover of $w$, can be derived from the characteristic function $w$ by defining

$$
v(S)=\max _{\left\{S_{k}\right\}} \sum_{k} w\left(S_{k}\right),
$$

where the maximum is taken over all partitions $\left\{S_{k}\right\}$ of $S$, called coalition structures. Such games $(N, v)$ are known as coalition structure games or partitioning games; see Aumann and Dreze (1974) where the concept was introduced, Kaneko and Wooders (1982) for conditions ensuring that collections of coalitions are strongly balanced (so that any game with those admissible coalitions has a nonempty core) and le Breton, Owen, and Weber (1992) for a discussion of games satisfying strong balancedness, and references in these papers.

Since we require feasibility of a payoff for the coalition of the whole, that is, the condition $x(N)=v(N)$ (see Section 2) our results do not make explicit any efficient underlying coalition structure, i.e., a coalition structure $\left\{S_{k}\right\}$ of $N$ such that $v(N)=\Sigma_{k} w\left(S_{k}\right)$. Our results, however, apply immediately to coalition structure games. The partnership structure associated with a point in the partnered core of $(N, v)$ informs us of the partnerships induced by the game $(N, w)$.

Proposition 1: Let $(N, w)$ be a game and let $(N, v)$ be the game in which $v$ is defined by (6.1).

(1.1) A payoff $x$ is a partnered payoff for the game $(N, w)$ if and only if $x$ is a partnered payoff for the game $(N, v)$.

(1.2) Given a partnered payoff $x$, let $\mathcal{P}_{x}^{w}$ denote the partnership structure (i.e., the collection of partnerships) induced by $x$ in the game $(N, w)$ and similarly let $\mathcal{P}_{x}^{v}$ be the partnership structure induced in $(N, v)$. Then $\mathcal{P}_{x}^{w}=\mathcal{P}_{x}^{v}$.

The proof follows from the fact that for any payoff $x$ the supporting coalitions induced by the game $(N, v)$ are unions of the supporting coalitions induced by the game $(N, w)$.

While the characteristic functions $v$ and $w$ induce the same partnerships, there is, however, a distinct difference between partnerships and coalitions. For example, consider a 2-person bargaining game, where there is a dollar to be divided between the two players 1 and 2 . The payoff $(.50, .50)$ is partnered with partnership $\{\{1,2\}\}$. The coalition $\{1,2\}$ can also realize the payoff $(1.00, .00)$, but the collection of supporting coalitions is not partnered and in this situation we do not call $\{1,2\}$ a partnership. 
To illustrate a further distinction between partnerships and efficient coalitions, suppose now that there are eight players, $\{1,2, \ldots, 8\}$. For this example, the eight players can be partitioned into four pairs, $p_{1}=\{1,2\}, p_{2}=\{3,4\}, p_{3}=\{5,6\}$ and $p_{4}=\{7,8\}$, and only distinct pairs of players are productive. ${ }^{6}$ Formally, define the characteristic function $w$ by

$$
\begin{gathered}
w(S)=2 \text { if } S=p_{i} \cup p_{j} \text { for some } i \neq j \text { and } \\
w(S)=0 \text { otherwise. }
\end{gathered}
$$

Observe that, using (6.1) to define $v$, an efficient coalition structure achieving $v(N)=4$ is given by

$$
\{\{1,2,3,4\},\{5,6,7,8\}\},
$$

or any other partition of the set of players into two coalitions, each consisting of two distinct pairs, $p_{i}$ and $p_{j}$. The core is given by

$$
\begin{gathered}
\left\{x \in R^{8}: x_{k} \geq 0 \text { for each player } \mathrm{k} \text { and for each pair } p_{i},\right. \\
\left.x\left(p_{i}\right)=1\right\} .
\end{gathered}
$$

The partnerships induced by any point in the relative interior of the core are the pairs $p_{1}, p_{2}, p_{3}$ and $p_{4}$ and the partnered core is

$$
\begin{gathered}
\left\{x \in R^{8}: x_{k}>0 \text { for each player } \mathrm{k} \text { and for each pair } p_{i},\right. \\
\left.x\left(p_{i}\right)=1\right\} .
\end{gathered}
$$

To realize a payoff in the partnered core the players in partnerships are inseparably united, while a number of different coalition structures (3, to be exact) are consistent with the achievement of any such outcome. This points to the distinction between partnerships and coalitions.

In the coalition structure literature (even in [1], [5], [6], [25]) no distinction is made between the concepts of coalitions and partnerships. Partnership refers to a closer link between players than that of membership in the same coalition.

Minimally partnered collections of coalitions appear to have special significance, and enable a strong distinction between models of economies. In terms of familiar economic examples, an economy with pure public goods has fully partnered Pareto-optimal outcomes and this is independent of the size of the economy.

\footnotetext{
${ }^{6}$ Vicky Barham suggested that the pairs may be pairs of automobile tires, with each pair a different size. Automobiles may have different sized tires in the front and in the rear, but the front wheels are both the same size and the back wheels are both the same size (and perhaps this is essential).
} 
Economies with local public goods, where the size of the groups of players required to achieve all gains to collective consumption and/or production are smaller than the number of players of each type, have Pareto-optimal outcomes that are minimally partnered. The competitive outcome of a replicated exchange economy is minimally partnered (Reny and Wooders (1996). All three sorts of economies are coalition structure economies. The feature of the existence of minimally partnered Pareto-optimal outcomes appears to be an important distinction.

\section{Other Literature Relating to the Partnership Property}

The partnership property first appeared in Maschler and Peleg $(1966,1967)$ and Maschler, Peleg, and Shapley (1971) in their study of the kernel of a cooperative game. We refer the reader to the original articles or to Owen (1982) or other texts in game theory for a definition of the kernel. Since the concern of Maschler and Peleg was "separating out" players, Maschler and Peleg call a partnered collection of sets a separating collection. They call two players $i$ and $j$ inseparable if, in our terminology, they are partners. Maschler and Peleg call a collection of sets completely separating if it is minimally partnered. Our choice of terminology follows Bennett (1983) and Bennett and Zame (1988) and is motivated by our desire to focus on coalition formation rather than the separation of players. The terms introduced by Maschler and Peleg are also appealing, as, from the viewpoint of core solutions, we may think of players who are partners as inseparable. A number of interesting relationships on balancedness and partnership are established in Maschler, Peleg, and Shapley (1971) including that a balanced collection of coalitions is partnered. This result, along with Lemma 3, lies at the heart of our proof of Theorem 1. Maschler, Peleg, and Shapley observe, however, that in general, the partnership property does not imply balancedness and that any set of six minimal winning coalitions in the 7-person projective game (c.f., von Neumann and Morgenstern (1953), p. 470) is minimally partnered but not even weakly balanced (i.e., balanced, but with some balancing weights in (4.3) possibly equal to zero).

The partnership property on the domain of undominated payoffs appears in Albers (1974,1979), Selten (1981), Bennett $(1980,1983)$, and Bennett and Zame (1988). In these papers the authors take the viewpoint that individuals, in bargaining over the distribution of payoff within a coalition, do not take into account the feasibility of the total demands of all participants in a game. A payoff $x$ for a game $(N, v)$ is defined as semi-stable (or an aspiration) if for each player $i$ there 
is a coalition $S$ containing $i$ such that $x(S)=v(S)$. The payoff $x$ is stable if it is semi-stable and partnered. Our definition of a partnered and undominated payoff implies that such a payoff is semi-stable (and thus, of course, stable). We note that our results continue to hold when we restrict the domain of payoffs to semi-stable demands (aspirations).

Besides its intuitive economic and game theoretic appeal and its mathematically interesting properties, the partnership property has additional motivation in non-cooperative game theory and in the theory of perfect competition. Selten (1981) provides an interesting example of the emergence of an undominated and partnered payoff (not necessarily feasible) in the description of a three person "divide the dollar" game. Bennett and Zame (1988) show that competitive payoffs of exchange economies with strictly convex and monotone preferences are partnered. Page and Wooders (1996) demonstrates that the Bennett-Zame result extends to economies with arbitrary consumption sets and possibly non-monotonicities. Page and Wooders also provide a Second Welfare Theorem for the partnered competitive equilibrium; every individually rational allocation is a partnered competitive equilibrium relative to some redistribution of endowments.

Reny and Wooders (1996) show that the partnered core of a balanced game without side payments is nonempty and thus provide a refinement of Scarf's Theorem (1967) on the nonemptiness of the core. Extending Theorem 4 of this paper, Reny and Wooders (1996) show that for any game with at most a countable number of points in its core, there is at least one core point which is minimally partnered. Reny and Wooders also provide an example of a game without side payments in which the relative interior of the core is nonempty but does not contain any payoffs with the partnership property. Thus, the character of the partnered core is quite distinct between games with and without side payments.

\section{References}

[1] Albers, W. (1974) "Zwei Logungskonzepte Fur Kooperative Mehrpersonspiele, Die Auf Anspruchsniveaus der Spieler Bastiern" OR-Verfahren (meth. Oper. Res) 21, 1-13.

[2] Albers, W. (1979) "Core and Kernel Variants Based on Imputations and Demand Profiles" in Game Theory and Related Topics, O. Moeschlin and D. Pallaschke, eds. North Holland, Amsterdam. 
[3] Aumann, R.J. and J. Dreze (1974) "Cooperative Games with Coalition Structures," International Journal of Game Theory 3, 217-237.

[4] Bennett, E. (1980) "Coalition Formation and Payoff Distribution in Cooperative Games," Ph. D Dissertation, Northwestern University (1980)".

[5] Bennett, E., (1983) "The Aspiration Approach to Predicting Coalition Formation and Payoff Distribution in Sidepayment Games," International Journal of Game Theory 12, 1-28.

[6] Bennett, E. and W.R. Zame (1988) "Bargaining in Cooperative games", International Journal of Game Theory 17, 279-300.

[7] le Breton, M., G. Owen, and S. Weber (1992) "Strongly Balanced Cooperative Games," International Journal of Game Theory 20, 419-427.

[8] Bondareva, O., (1963) "Some Applications of Linear Programming Methods to the Theory of Cooperative Games," Problemy Kybernetiki 10, 119-139 (in Russian).

[9] Kaneko, M. and M.H. Wooders (1982) "Cores of Partitioning Games," Mathematical Social Sciences 3, 313-327.

[10] Kannai, Y. and M. Wooders (2000) "A further extension of the KKMS Theorem," Mathematics of Operations Research 25, 539-551.

[11] Maschler, M. and B. Peleg (1966) "A Characterization, Existence Proof and Dimension Bounds for the Kernel of a Game," Pacific Journal of Mathematics 18, 289-328.

[12] Maschler, M. and B. Peleg (1967) "The structure of the kernel of a cooperative game," SIAM Journal of Applied Mathematics 15, 569-604.

[13] Maschler, M., B. Peleg, and L.S. Shapley (1971) "The Kernel and Bargaining Set for Convex Games," International Journal of Game Theory 1, 73-93.

[14] Moldovanu, B. and E. Winter (1994) "Consistent Demands for Coalition Formation in Games," in Essays in Game Theory in Honor of Michael Maschler, ed. Nimrod Megiddo. Springer-Verlag, 129-140.

[15] Page, F.H. Jr. and M. Wooders (1996) "The Partnered Core and the Partnered Competitive Equilibrium," Economics Letters 52 (1996) 143-152. 
[16] von Neumann, J. and O. Morgenstern (1953) The Theory of Games, Princeton University Press.

[17] Owen, G. (1982) Game Theory, Academic Press, New York.

[18] Peleg, B. (1968) "On Minimal Separating Collections," Proceedings of the American Mathematical Society 19, 1-3.

[19] Reny, P.J. and M. Wooders (1996) "The Partnered Core of a Game Without Side Payments," Journal of Economic Theory 70, 298-311.

[20] Reny, P.J. and M. Wooders (1998) "An Extension of the KKMS Theorem," Journal of Mathematical Economics 29,125-134.

[21] Reny, P.J., E. Winter and M. Wooders (1993) "The Partnered Core of a Game With Side Payments," Hebrew University Center for Rationality Working Paper 33; University of Bonn Sonderforschungsbereich 303, in the German National Library, OCLC 7551219; 1University of Western Ontario Research Report ISBN 0771415893;

[22] Rockafellar, R.T. (1970) Convex Analysis, Princeton University Press, Princeton, New Jersey.

[23] Scarf, H.E. (1967) "The Core of an n-person Game," Econometrica 35, 50-67.

[24] Schrijver, A. (1990) Theory of Linear and Integer Programming, Wiley Interscience Series in Discrete Mathematics and Optimization.

[25] Selten, R. (1981) "A Noncooperative Model of Characteristic Function bargaining," in Essays in Game Theory and Mathematical Economics in Honor of Oskar Morgenstern, V. Boehm and H. Nacktkamp (eds.), in Gesellschaft, Recht, Wirtschaft, Band 4, Wissenschaftsverlag Bibliographisches Institute. Mannheim-Wein-Zurich, 131-151.

[26] Shapley, L.S. (1967) "On Balanced Sets and Cores," Navel Research Logistics Quarterly 14, 463-460.

[27] Winter, E. (1989) "An Axiomatization of the Stable and Semistable Demand Vectors by the Reduced Game Property" Discussion Paper No. A-254, The University of Bonn. 
[28] Wooders, M. (1994) "Large Games and Economies with Effective Small Groups," in Game-Theoretic Methods in General Equilibrum Analysis, eds. J-F. Mertens and S. Sorin, Kluwer Academic Publishers Dordrecht/Boston/London 145-206. 